\title{
Editorial
}

European

Thyroid Journal
Eur Thyroid J 2018;7:165-166

DOI: $10.1159 / 000491585$
Received: June 26, 2018

Accepted: June 26, 2018

Published online: July 19, 2018

\section{Reflections on the Management of Graves' Hyperthyroidism}

\author{
Chantal Daumerie ${ }^{a}$ Jacques Orgiazzi ${ }^{b}$ \\ a Service Endocrinologie, Université Catholique de Louvain, Cliniques Universitaires Saint-Luc, Brussels, Belgium; \\ ${ }^{\mathrm{b}}$ Hospices Civils de Lyon, Université Claude-Bernard Lyon 1, Lyon, France
}

Hyperthyroid Graves' disease (GD) is an autoimmune thyroid disease caused by stimulating thyrotropin (TSH) receptor antibodies (TRAb). As early as the 1940s, the three components of the current therapeutic armamentarium for hyperthyroidism were already available: antithyroid drugs and radioiodine treatment as recent innovations, and thyroidectomy since the early part of the century. For years, clinical research has tried to improve the dilemma: conservative medical restoration of euthyroidism - with a 50\% risk of subsequent relapse - or removal/ radical inactivation of thyroid tissue at the expense of another disease, a definitive one, hypothyroidism. Finally, after years, it became accepted that, once selected, "radical" treatment should be really radical, aiming at the definite eradication of hyperthyroidism. For radioiodine treatment, the concept of "ablative" dose was introduced instead of the vain search for doses aiming at euthyroidism. For thyroidectomy, the rule of "total" or "near-total" ablation would avoid the recurrences observed after subtotal thyroidectomy. In the meantime, thyroid function tests, TRAb assay, and imaging procedures have been improved and side effects of antithyroid drugs have been better characterized. Also, current research in the immunology of GD and the interaction of TRAb with the TSH receptor raises hope that therapeutic means addressing the etiopathogenesis of the disease are on their way. Another complexity of the management results from the kaleidoscopic aspects of the disease according to the various patients' presentations: age, physiological conditions (e.g., pregnancy), and presence of orbitopathy and other serious complications. Graves' hyperthyroidism needs to be diagnosed and treated as soon as possible to insure a fair quality of life and prevent long-term complications. To provide patients with the best quality care, an overwhelming amount of recent scientific information has to be mastered. To that end, guidelines have been issued by scientific societies. However, physicians may sometimes elect, on clinical evidence, not to follow some of the recommendations.

This issue of the European Thyroid Journal publishes the European Thyroid Association (ETA) 2018 Guidelines for the management of Graves' hyperthyroidism sponsored by the ETA guideline committee. The 50 recommendations have been elaborated, with the coordination of Pr. George Kahaly, by thyroidologists from Western European countries, each a recognized expert in their own subspecialty, and all engaged in clinical practice and research.

\section{KARGER}

E-Mail karger@karger.com

www.karger.com/etj (c) 2018 European Thyroid Association

Published by S. Karger AG, Basel
Jacques Orgiazzi

Hospices Civils de Lyon, Université Claude-Bernard Lyon 1

Quai des Célestins

FR-69002 Lyon (France)

E-Mail jacques.orgiazzi@chu-lyon.fr 
Once GD hyperthyroidism is suspected, and then confirmed by biochemical tests and TRAb determination, the next diagnostic tests are dependent on available expertise and resources and therefore, for instance, imaging procedures may differ between places. The guidelines recommend thyroid ultrasound as part of the initial work-up, especially in the nodular thyroid gland. However, thyroid ultrasound examination performed by an experienced radiologist using a high-resolution Doppler ultrasonography machine may not be available everywhere and, even if so, may not be standard. So, in the absence of a prompt result of a TRAb assay, thyroid technetium scintigraphy with determination of thyroid uptake of the tracer remains an easy approach for the elucidation of the type of hyperthyroidism. Operator independent, it is also diagnostic of autonomous adenoma or toxic multinodular goiter and of iodine contamination. As to the treatment, the guidelines state that antithyroid drugs are usually selected as the first-line treatment. Patients may be treated according to the titration or the block-and-replace regimen. Some physicians start with the titration regimen and subsequently shift to the block-and-replace one. While recurrence rates of Graves' hyperthyroidism are reported to be similar in the two regimens, none seems to have specific advantages except for the lower prevalence of antithyroid drug side effects in the titration regimen [1]. However, the studies available are retrospective and it is surprising that no prospective clinical randomized trial has been reported. It is well accepted that a stable euthyroid status is primordial for avoiding complications such as the development of Graves' orbitopathy (GO) but this has never been demonstrated by clinical studies. As clearly presented in the guidelines, management of GD when GO is present requires insight and experience. Prompt restoration and stabilization of euthyroidism are a priority in patients with GO. It is considered that GD is a long-lasting disease, with an arguable type of treatment strategy which may durably affect the patient's life. Con- sequently, it is clearly stated in the American Thyroid Association guidelines for hyperthyroidism published in 2016, and usually implemented, that "once the diagnosis has been made, the treating physician and patient should discuss each of the treatment options, including the logistics, benefits, expected speed of recovery, drawbacks, potential side effects, and cost" [2]. Obviously, socio-professional obligations, lifestyle, and personal preferences are also an important part of the patient's contribution to the final therapeutic decision and should be accounted for. The completion of the 12- to 18-month antithyroid drug course, when discontinuation of treatment is considered, is a crucial phase which requires a specific patient-physician interaction. Risk of relapse should be confronted with the relevant risk factors discussed initially and according to the current clinical and biological status. TRAb level is infallibly predictive of relapse if elevated, while a low level is only weakly associated with remission. Occurrence of relapse may be considered a point of major disappointment by the patient. At this point, three options may be considered: a second course of antithyroid drug, continuation of the antithyroid drug at low dose if appropriate, or radical treatment - either total thyroidectomy or radioactive iodine. Another important observation is that GD, and not only GO, significantly affects the quality of life. Furthermore, studies have suggested this impact to be long-lasting and even to outlast the treatment period [3]. These domains are of timely interest.

It may be surprising that, in these complete guidelines, only 18/50 recommendations are labeled as "strong" and supported by high-quality papers. Designing randomized clinical studies in order to validate some of the weak recommendations should be considered. Indeed, clinical research is still active. Finally, in any difficult situation, Hippocrates' motto "primum non nocere" should prevail. Therefore, these difficult GD patients should be referred to expert centers with a high volume of such patients.

\section{References}

1 Vaidya B,Wright A, Shuttleworth J, et al: Block and replace regime versus titration regime of antithyroid drugs for the treatment of Graves' disease: a retrospective observational study. Clin Endocrinol 2014;81:610-613.

2 Ross DS, Burch HB, Cooper DS, et al: 2016 American Thyroid Association Guidelines for Diagnosis and Management of Hyperthy- roidism and Other Causes of Thyrotoxicosis. Thyroid 2016;10:1343-1421.

3 Abraham-Nordling M, Törring O, Hamberger B, et al: Graves' disease: a long-term quality-of-life follow up of patients randomized to treatment with antithyroid drugs, radioiodine, or surgery. Thyroid 2005;151:12791286. 